\title{
Multidimensional Trajectory Tracking for Numerically Stiff Independent Metering System
}

\author{
Goran Stojanoski , Dimitar Ninevski , Gerhard Rath, and Matthew Harker* \\ Chair of Automation, University of Leoben, Leoben, Austria \\ *Faculty of Engineering and Applied Science, Ontario Tech University, Ontario, Canada \\ E-mail: \{goran.stojanoski, dimitar.ninevski, gerhard.rath\}@unileoben.ac.at; matthew.harker@ontariotechu.ca
}

\begin{abstract}
This paper presents a new approach for solving an optimal control problem in a hydraulic system, using a variational calculus method. It uses a path tracking method of two different states with different units and of different magnitude. To ensure the uniqueness of the solution, two regularization terms were introduced, whose influence is regulated by regularization parameters. The system of differential equations, obtained from the Euler-Lagrange equations of the variational problem, was solved by a mass matrix method and discretized with linear differential operators at the interstitial points for numerical stability. This enabled the calculation of the control variables, despite the stiffness of the numerical problem. The results obtained show an energyefficient performance and no oscillations. Finally, a Simulink model of the hydraulic system was created in which the calculated control variables were inserted as feedforward inputs, to verify the results.
\end{abstract}

Keywords: Hydraulics, Optimal control, Independent metering, Euler-Lagrange.

\section{Introduction}

The theory of optimal control has existed for several centuries [1]. When applied to a hydraulic system, it dampens all vibrations at the end of the motion and still achieves the desired values [2]. However, due to the extremely nonlinear dynamics its use in hydraulics is a challenge [3]. With the increasing demands for accuracy and performance in mining, especially for tunnel boring machines, the demand for new methods is also increasing. In addition, these new methods must deliver energy efficient control results and reduce the operating costs of the entire process. The new state of the art independent metering valves, which are an essential part of these systems, offer very flexible control strategies [4]. Since the valves move much faster than the natural frequency of the load system, the ODEs to be solved become numerically very stiff [5]. For this reason these systems cannot be solved with conventional solvers [6]. Rath in [5] shows that the use of exponential matrix results in unstable solutions for these type of systems. In this paper, the mass matrix method and interstitial derivatives are used to compute a stable numerical solution. Since tunnel boring machines have a given profile which has to be cut, optimal path tracking methods are suitable for these types of systems. Compared to conventional methods, optimal path tracking is often used for the navigation of mining equipment in mines. It increases efficiency and reduces working time, which in turn increases the safety of the process [7] [8]. In most cases, there is a given path that the system must follow [9] [10]. One of the methods that is frequently used is model predictive control (MPC) [11] [12] because it can take the constraints of the system into account [13]. Wang in [14] improves the efficiency of the ant colony algorithm to find the optimal path for barrier environments of varying complexity. Path tracking is also frequently used for control of multi-dimensional hydraulic manipulators. In [15], the trajectory of a one-armed hydraulic manipulator is tracked by a digital hydraulic system. Kalaiarassan also shows that the 5-bit digital flow control unit performs much better than the 4-bit system. Rudolfsen [16] solves the kinematics of a crane for operation in the vertical plane. He uses the inverse to compensate and identify the non-linearity of the static dead zone input signals. A global least 
squares method is used in [17] to determine the optimal control input for multi-dimensional path tracking of a hydraulic crane driven by electric drives. In [18], a new control law based on the sliding perturbation observer (SPO) is designed. Here the SPO is used to eliminate all disturbances that come from the environment, dynamic uncertainties and modeling errors. Chin in [19] presents a new type of contour tracking control which uses the force calculation for hydraulic parallel manipulators. Zhang in [20] uses the D-H (Denavit - Hartenberg) method to set the coordinates for the path of a hydraulic excavator. On the other hand, Kang [21] uses a PD controller with dead zone compensation to track the three-dimensional path. However, all of the above methods focus on tracking one or more parameters which have the same unit and magnitude and, in most cases, the position of the given system is tracked. Tunnel boring machines have very complex hydraulic circuits. In the case presented here, the valve is equipped with pressure and flow controllers that actuate the system. Since the external forces are very high, the set pressure value at the pressure controller is usually very high. This will increase the mechanical stiffness of the hydraulic system which in turn increases the mechanical stiffness of the overall system. However, the PID controllers normally used in these valves show a very oscillating behavior when the value of the pressure changes. In [22] it is shown that different pressure values during a motion can lead to an improved energy performance of these systems. The method proposed in this paper, which is based on the theory of optimal control, solves the tracking problem as a boundary value problem by means of variational calculus. The method is then applied to a simulated model of the actual system in order to show its potential.

The contributions of this paper are:

1. A novel linearized state space model for the system presented in fig. 1 is derived. For the first time the embedded valve controllers (flow and pressure) and the position controller (responsible for the position of the large mass) are included in the state space system.

2. A new method for multidimensional trajectory tracking of parameters with different units and magnitudes is presented on the basis of calculus of variations.

3. The mass matrix method and interstitial derivatives are used to calculate a stable numerical solution for this stiff numerical problem.

4. A simulation in Matlab Simulink is performed to verify the proposed control scheme.

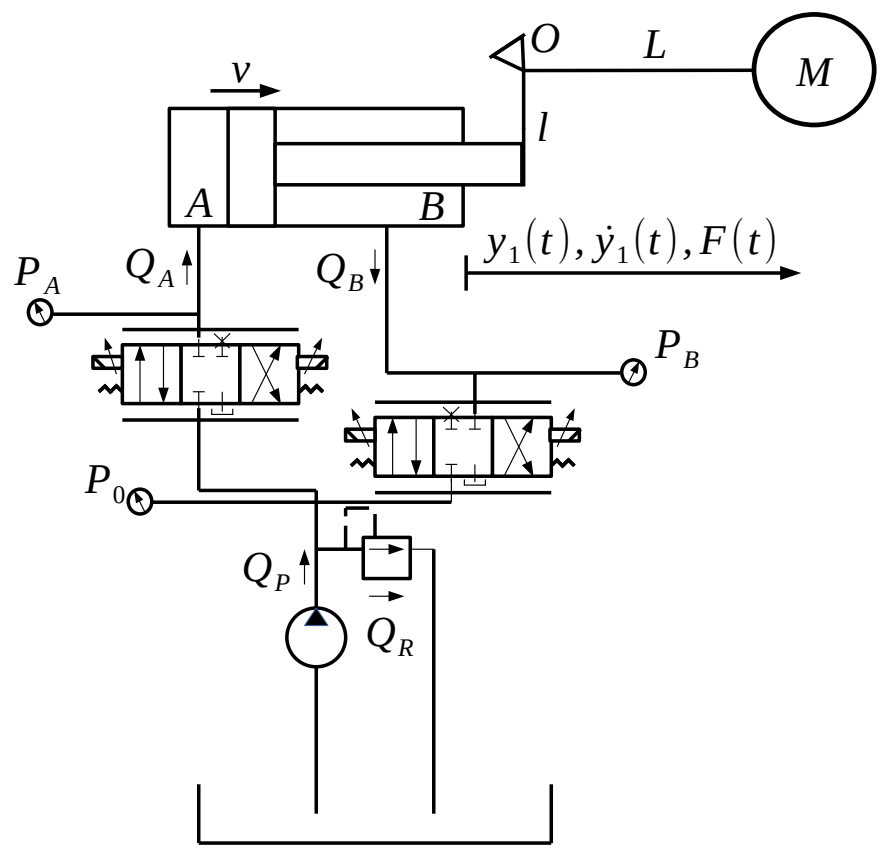

Figure 1: Simplified model of the hydraulic and mechanical system. The mechanical system is position controlled. The hydraulic system consist of two independent metering valves which are flow controlled (piston side) and pressure controlled (rod side). Auxiliary components, like pressure compensators and load sensing are not shown in this figure. 
The results show that the system can always achieve the desired paths for position and pressure. Furthermore, despite the pressure changes significantly, there are no oscillations present during the motion.

\section{SYSTEM MODEL}

\subsection{Mechanical System}

The mechanical system consists of a large mass rotating around a point $\mathrm{O}$ (fig. 1) with a lever of length $L$. The hydraulic cylinder exerts the force $F$ on a smaller lever of length $l$. If we assume that the system makes small movements around the fulcrum $\mathrm{O}$, the effective mass of the system is

$$
m=\left(\frac{l}{L}\right)^{2} M
$$

If the position of the mass $m$ in fig. 1 is labeled as $y_{1}$, the equation of motion will have the form

$$
m \ddot{y_{1}}=P_{A} A_{A}-P_{B} A_{B}-b \dot{y_{1}} \text {. }
$$

\subsection{Hydraulic System}

The hydraulic part of the system consists of a hydraulic pump, two independent metering valves and a hydraulic actuator. In this system the friction consists of several components. The oil flow through the valve orifice depends on the pressure which can cause damping. In addition, the movement of the load and the steel structure also contribute to the overall friction. The hydraulic cylinder has a highly non-linear friction behavior. A complete mathematical model of friction includes the Stribeck and stiction effect as well as Coulomb and viscous friction [23]. In the actual machine however, the greatest contribution comes from the rotating load that performs a cutting operation. Therefore, in eq. (2) the friction is assumed to be viscous with coefficient $b$.

\subsection{Dynamics of the system}

The displacement of the mechanical system is controlled by a position controller which actuates the two independent metering valves. The valves offer different operating modes for different load scenarios [4]. In this case it is assumed that the load on the system is passive when the movement is positive. This means that the actuating side of the system is flow controlled and the rear side is pressure controlled. The flows supplied by the valves are a function of the spool movement $x_{v}$ and the pressures $P_{A}, P_{B}$ [24],

$$
Q_{A}=C_{q_{1}} x_{v_{1}}-C_{p_{1}} P_{A}, \quad Q_{B}=C_{q_{2}} x_{v_{2}}+C_{p_{2}} P_{B}
$$

where $C_{q 1}, C_{q 2}, C_{p 1}, C_{p 2}$ are the valve linearization coefficients and $x_{v_{1}}, x_{v_{2}}$ are the valves' spool positions. If eq. (3) is combined with the equations for the flow through the cylinder [25] the pressure equations are obtained:

$$
P_{A}=\frac{C_{q_{1}} x_{v_{1}}-A_{A} v}{\frac{V_{A}}{\beta} s+C_{p_{1}}}, \quad P_{B}=\frac{A_{B} v-C_{q_{2}} x_{v_{2}}}{\frac{V_{B}}{\beta} s+C_{p_{2}}},
$$

where $\beta$ is the bulk modulus of the oil, $V_{A}$ and $V_{B}$ the volumes and $A_{A}$ and $A_{B}$ the areas of the cylinder chambers. After deriving the linearized equations for the valve actuator system eq. (4), the controllers of the system can be applied. The independent metering valves presented in fig. 1 react much faster compared to the natural frequency of the load system. For this reason, no flow controller is implemented into the system in fig. 2. Here $k_{A}$ and $k_{B}$ are the proportional parts of the PID controllers for both sides. The system shown in fig. 2 is governed in state space form with mass matrix $\Pi$ by the following equations

$$
\Pi \dot{x}(t)=A x(t)+B u(t)
$$

where,

$$
\Pi=\left[\begin{array}{cccc}
\frac{1}{\beta} & 0 & 0 & 0 \\
0 & \frac{1}{\beta} & 0 & 0 \\
0 & 0 & 1 & 0 \\
0 & 0 & 0 & m
\end{array}\right], x=\left[\begin{array}{c}
P_{A} \\
P_{B} \\
y_{1} \\
v
\end{array}\right], u=\left[\begin{array}{c}
y_{\text {set }} \\
P_{B s e t}
\end{array}\right]
$$




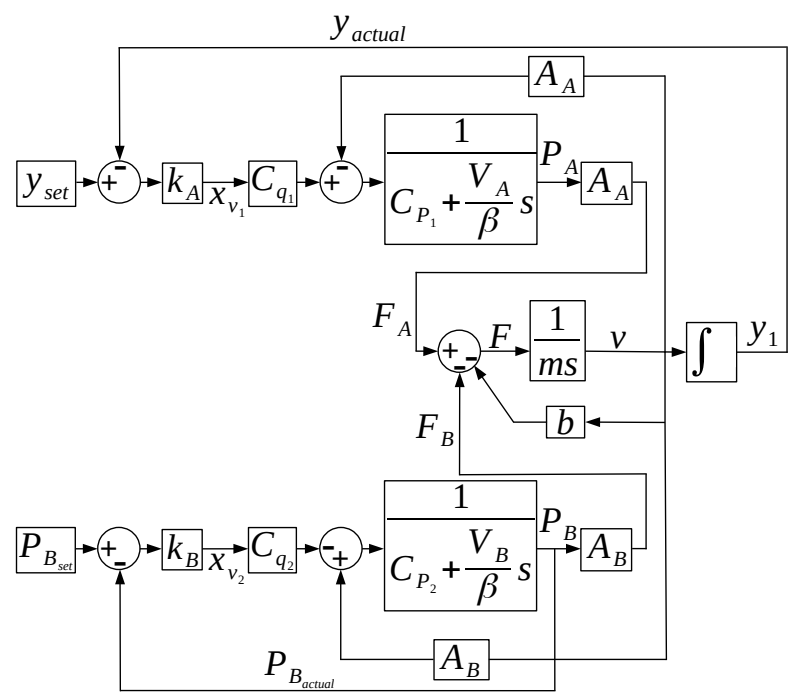

Figure 2: State of the art block diagram for the system presented in fig. 1. The system is controlled on both sides, where $y_{\text {set }}$ and $P_{B_{\text {set }}}$ are the desired position and pressure values. Here $k_{A}$ and $k_{B}$ are the proportion parts of the PID controllers for both sides accordingly.

$$
\begin{gathered}
A=\left[\begin{array}{cccc}
-C_{p_{1} \frac{\beta}{V_{A}}} & 0 & -k_{A} C_{q_{1}} \frac{\beta}{V_{A}} & -A_{A} \frac{\beta}{V_{A}} \\
0 & -\left(k_{B} C_{q_{2}}+C_{p_{2}}\right) \frac{\beta}{V_{B}} & 0 & -A_{B} \frac{\beta}{V_{B}} \\
0 & 0 & 0 & 1 \\
\frac{A_{A}}{m} & -\frac{A_{B}}{m} & 0 & -\frac{b}{m}
\end{array}\right] \\
B=\left[\begin{array}{ccc}
k_{A} C_{q_{1}} \frac{\beta}{V_{A}} & 0 \\
0 & -k_{B} C_{q_{2}} \frac{\beta}{V_{B}} \\
0 & 0 \\
0 & 0
\end{array}\right]
\end{gathered}
$$

In eq. (6, eq. (7) and eq. (8) the value of the bulk modulus $\beta$ and the mass $m$ are on both sides of the equations. Since $\beta$ and $m$ have very high values, a fraction of these values was stored in the mass matrix to ensure a numerically stable solution.

\section{Solution of the problem}

The path tracking problem can be formulated as determining the input vector $u$, so that a desired output of the system is achieved. This problem can be formulated in terms of variational calculus, as finding the optimal input vector $u$ for which the cost function

$$
\frac{\mu_{1}^{2}}{2} \int_{t_{0}}^{t_{f}}\left(x_{2}(t)-\xi_{2}(t)\right)^{2} \mathrm{dt}+\frac{\mu_{2}^{2}}{2} \int_{t_{0}}^{t_{f}}\left(x_{3}(t)-\xi_{1}(t)\right)^{2} \mathrm{dt}
$$

is minimized. Note that because of the way the vector $u$ is defined, $x_{2}$ tracks $\xi_{2}$ and $x_{3}$ tracks $\xi_{1}$. The integrals quantify the least-squares differences between the states $x_{2}(t), x_{3}(t)$ and the desired paths $\xi_{2}(t)$, $\xi_{1}(t)$ respectively. Since the states $x_{2}(t), x_{3}(t)$ are measured in different units of different magnitude, $\mathrm{Pa}$ and $\mathrm{m}$ respectively, a normalization of the least squares differences is necessary. This is done with the parameters $\mu_{1}, \mu_{2}$. The previous equation can be rewritten as

$$
\frac{\mu_{1}^{2}}{2} \int_{t_{0}}^{t_{f}}\left(e_{2}^{\mathrm{T}} x(t)-\xi_{2}(t)\right)^{2} \mathrm{dt}+\frac{\mu_{2}^{2}}{2} \int_{t_{0}}^{t_{f}}\left(e_{3}^{\mathrm{T}} x(t)-\xi_{1}(t)\right)^{2} \mathrm{dt},
$$

where $e_{2}=\left[\begin{array}{llll}0 & 1 & 0 & 0\end{array}\right]^{\mathrm{T}}$ and $e_{3}=\left[\begin{array}{llll}0 & 0 & 1 & 0\end{array}\right]^{\mathrm{T}}$ are coordinate unit vectors. In order to get a unique solution, a regularization parameter is also necessary [26], which in the case discussed here looks as follows:

$$
\frac{\mu_{3}^{2}}{2} \int_{t_{0}}^{t_{f}} \dot{u}^{\mathrm{T}}(t) \dot{u}(t) \mathrm{dt}+\frac{\mu_{4}^{2}}{2} \int_{t_{0}}^{t_{f}} \ddot{u}^{\mathrm{T}}(t) \ddot{u}(t) \mathrm{dt} .
$$


Finally, the functional which needs to be minimized will have the following form:

$$
\begin{aligned}
& J(x(t), u(t), \lambda(t))= \\
& \frac{\mu_{1}^{2}}{2} \int_{t_{0}}^{t_{f}}\left(e_{2}{ }^{\mathrm{T}} x(t)-\xi_{2}(t)\right)^{2} \mathrm{dt}+\frac{\mu_{2}^{2}}{2} \int_{t_{0}}^{t_{f}}\left(e_{3}^{\mathrm{T}} x(t)-\xi_{1}(t)\right)^{2} \mathrm{dt} \\
& +\frac{\mu_{3}^{2}}{2} \int_{t_{0}}^{t_{f}} \dot{u}^{\mathrm{T}}(t) \dot{u}(t) \mathrm{dt}+\frac{\mu_{4}^{2}}{2} \int_{t_{0}}^{t_{f}} \ddot{u}^{\mathrm{T}}(t) \ddot{u}(t) \mathrm{dt} \\
& -\int_{t_{0}}^{t_{f}} \lambda^{\mathrm{T}}(t)(\Pi \dot{x}(t)-A x(t)-B u(t)) \mathrm{dt}
\end{aligned}
$$

In eq. (12) only the ratio $\mu_{1}: \mu_{2}: \mu_{3}: \mu_{4}$ is relevant, thus one of the parameters can be set arbitrarily. That is why $\mu_{1}$ is set to be 1 . The Euler-Lagrange equations [27] for this variational problem are as follows:

$$
\begin{aligned}
& e_{2} e_{2}{ }^{\mathrm{T}} x-e_{2} \xi_{2}+\mu_{2}^{2} e_{3} e_{3}{ }^{\mathrm{T}} x-\mu_{2}^{2} e_{3} \xi_{1}+A^{\mathrm{T}} \lambda+\Pi^{\mathrm{T}} \dot{\lambda}=0 . \\
& B^{\mathrm{T}} \lambda-\mu_{3}^{2} \ddot{u}+\mu_{4}^{2} u^{(4)}=0 \\
& \Pi \dot{x}(t)+A x(t)+B u(t)=0 .
\end{aligned}
$$

From these three equations the following system of differential equations is derived:

$$
\begin{aligned}
& \Pi^{\mathrm{T}} \dot{\lambda}=-E_{23} x+e_{2} \xi_{2}+\mu_{2}^{2} e_{3} \xi_{1}-A^{\mathrm{T}} \lambda \\
& u^{(4)}=-\frac{1}{\mu_{4}^{2}} B^{\mathrm{T}} \lambda+\frac{\mu_{3}^{2}}{\mu_{4}^{2}} \ddot{u} \\
& \Pi \dot{x}(t)=A x(t)+B u(t)
\end{aligned}
$$

where

$$
E_{23}=e_{2} e_{2}^{\mathrm{T}}+\mu_{2}^{2} e_{3} e_{3}^{\mathrm{T}}
$$

These equations can be written compactly in matrix form as:

$$
\Pi_{1} \dot{\gamma}(t)-V \gamma(t)-W \xi(t)=0
$$

where

$$
\begin{aligned}
\Pi_{1} & =\left[\begin{array}{cccccc}
\Pi & 0 & 0 & 0 & 0 & 0 \\
0 & \Pi^{\mathrm{T}} & 0 & 0 & 0 & 0 \\
0 & 0 & I & 0 & 0 & 0 \\
0 & 0 & 0 & I & 0 & 0 \\
0 & 0 & 0 & 0 & I & 0 \\
0 & 0 & 0 & 0 & 0 & I
\end{array}\right], \gamma(t)=\left[\begin{array}{c}
x(t) \\
\lambda(t) \\
u(t) \\
\dot{u}(t) \\
\ddot{u}(t) \\
u^{(3)}(t)
\end{array}\right], \xi=\left[\begin{array}{c}
\xi_{1} \\
\xi_{2}
\end{array}\right], \\
V & =\left[\begin{array}{cccccc}
A & 0 & B & 0 & 0 & 0 \\
-E_{23} & -A^{\mathrm{T}} & 0 & 0 & 0 & 0 \\
0 & 0 & 0 & I & 0 & 0 \\
0 & 0 & 0 & 0 & I & 0 \\
0 & 0 & 0 & 0 & 0 & I \\
0 & -\frac{1}{\mu_{4}^{2}} B^{\mathrm{T}} & 0 & 0 & \frac{\mu_{3}^{2}}{\mu_{4}^{2}} & 0
\end{array}\right], W=\left[\begin{array}{cc}
0 & 0 \\
e_{2} & \mu_{2}^{2} e_{3} \\
0 & 0 \\
0 & 0 \\
0 & 0 \\
0 & 0
\end{array}\right] .
\end{aligned}
$$

\subsection{Numerical Solution}

Transposing eq. (16) one gets,

$$
\dot{\gamma}^{\mathrm{T}}(t) \Pi_{1}^{\mathrm{T}}-\gamma^{\mathrm{T}}(t) V^{\mathrm{T}}-\xi^{\mathrm{T}}(t) W^{\mathrm{T}}=0
$$

Now, for this system, the input vector is known (because it consists of the desired output) and can be solved numerically by discretizing with the methods described in [28]. The methods can be described as follows: note first that any state can be discretized directly as a vector,

$$
\gamma_{k}=\left[\begin{array}{llll}
\gamma_{k}\left(s_{0}\right) & \gamma_{k}\left(s_{1}\right) & \ldots & \gamma_{k}\left(s_{f}\right)
\end{array}\right]^{\mathrm{T}}
$$


or, if the discretization is done at the interstitial points (the points $t_{i}$, between the samples $s_{i}$ ), one gets

$$
\begin{gathered}
J_{0}=\frac{1}{16}\left[\begin{array}{c}
\gamma_{k}\left(t_{1}\right) \\
\gamma_{k}\left(t_{2}\right) \\
\vdots \\
\gamma_{k}\left(t_{f}\right)
\end{array}\right]=J_{0}\left[\begin{array}{c}
\gamma_{k}\left(s_{0}\right) \\
\gamma_{k}\left(s_{1}\right) \\
\vdots \\
\gamma_{k}\left(s_{f}\right)
\end{array}\right] \\
\left.\begin{array}{cccccccccc}
5 & 15 & -5 & 1 & 0 & \ldots & 0 & 0 & 0 & 0 \\
-1 & 9 & 9 & -1 & 0 & \ldots & 0 & 0 & 0 & 0 \\
0 & -1 & 9 & 9 & -1 & \ldots & 0 & 0 & 0 & 0 \\
\vdots & \vdots & \vdots & \vdots & \vdots & \ddots & \vdots & \vdots & \vdots & \vdots \\
0 & 0 & 0 & 0 & 0 & \ldots & -1 & 9 & 9 & -1 \\
0 & 0 & 0 & 0 & 0 & \ldots & 1 & -5 & 15 & 5
\end{array}\right]
\end{gathered}
$$

Using this, any vector of states $\gamma$ can be discretized as a matrix in the following way

$$
\Gamma^{T}=\left[\begin{array}{c}
\gamma_{1}^{\mathrm{T}} \\
\gamma_{2}^{\mathrm{T}} \\
\vdots \\
\gamma_{n}^{\mathrm{T}}
\end{array}\right]=\left[\begin{array}{cccc}
\gamma_{1}\left(s_{0}\right) & \gamma_{1}\left(s_{1}\right) & \ldots & \gamma_{1}\left(s_{f}\right) \\
\gamma_{2}\left(s_{0}\right) & \gamma_{2}\left(s_{1}\right) & \ldots & \gamma_{2}\left(s_{f}\right) \\
\vdots & \vdots & \ddots & \vdots \\
\gamma_{n}\left(s_{0}\right) & \gamma_{n}\left(s_{1}\right) & \ldots & \gamma_{n}\left(s_{f}\right)
\end{array}\right] J_{0}{ }^{\mathrm{T}}
$$

Additionally, the derivative of a state can be discretized as

$$
\dot{\gamma}_{k} \approx D \gamma_{k},
$$

where $D$ is a differentiation matrix, with the following form

$$
D=\frac{1}{24 h}\left[\begin{array}{cccccccccc}
-23 & 21 & 3 & -1 & 0 & \ldots & 0 & 0 & 0 & 0 \\
1 & -27 & 27 & -1 & 0 & \ldots & 0 & 0 & 0 & 0 \\
0 & 1 & -27 & 27 & -1 & \ldots & 0 & 0 & 0 & 0 \\
\vdots & \vdots & \vdots & \vdots & \vdots & \ddots & \vdots & \vdots & \vdots & \vdots \\
0 & 0 & 0 & 0 & 0 & \ldots & 1 & -27 & 27 & -1 \\
0 & 0 & 0 & 0 & 0 & \ldots & 1 & -3 & -21 & 23
\end{array}\right]
$$

where it is assumed that the discretization is done uniformly with step size $h$. Hence, a vector of first derivatives of states can be discretized as the following matrix

$$
\Gamma^{T}=\left[\begin{array}{c}
\dot{\gamma}_{1}^{\mathrm{T}} \\
\dot{\gamma}_{2}^{\mathrm{T}} \\
\vdots \\
\dot{\gamma}_{n}^{\mathrm{T}}
\end{array}\right]=\left[\begin{array}{cccc}
\gamma_{1}\left(s_{0}\right) & \gamma_{1}\left(s_{1}\right) & \ldots & \gamma_{1}\left(s_{f}\right) \\
\gamma_{2}\left(s_{0}\right) & \gamma_{2}\left(s_{1}\right) & \ldots & \gamma_{2}\left(s_{f}\right) \\
\vdots & \vdots & \ddots & \vdots \\
\gamma_{n}\left(s_{0}\right) & \gamma_{n}\left(s_{1}\right) & \ldots & \gamma_{n}\left(s_{f}\right)
\end{array}\right] D^{\mathrm{T}}
$$

Finally, the discretized form of eq. (18) is

$$
D \Gamma \Pi_{1}^{\mathrm{T}}-J_{0} \Gamma V^{\mathrm{T}}-J_{0} \Xi W^{\mathrm{T}}=0 .
$$

where $\Gamma$ and $\Xi$ are the matrices derived from the discretization of $\gamma$ and $\xi$ respectively. For more details, see [28,29]. Finally vectorizing the last equation, one gets

$$
\left(\Pi_{1} \otimes D-V \otimes J_{0}\right) \operatorname{vec}(\Gamma)=\left(W \otimes J_{0}\right) \operatorname{vec}(\Xi)
$$

which is a linear system of equations and can be solved using standard methods for linear systems of equations, along with appropriate constraints (SVD, QR decomposition). In order to get a unique solution, an appropriate number of initial and final conditions need to be defined. From the system of 16 differential equations eq. (18), a 
total of 16 initial and boundary conditions are given. ${ }^{1}$

$$
\begin{array}{lll}
\gamma_{1}\left(t_{0}\right)=0 & & \\
\gamma_{1}\left(t_{f}\right)=0 & \gamma_{4-8}\left(t_{0}\right)=0 & \gamma_{10}\left(t_{0}\right)=\xi_{1}\left(t_{0}\right) \\
\gamma_{2}\left(t_{0}\right)=\xi_{2}\left(t_{0}\right) & \gamma_{4-8}\left(t_{f}\right)=0 & \gamma_{10}\left(t_{f}\right)=\xi_{1}\left(t_{f}\right) \\
\gamma_{2}\left(t_{f}\right)=\xi_{2}\left(t_{f}\right) & \gamma_{9}\left(t_{0}\right)=\xi_{2}\left(t_{0}\right) & \gamma_{11-16}\left(t_{0}\right)=0 \\
\gamma_{3}\left(t_{0}\right)=\xi_{1}\left(t_{0}\right) & \gamma_{9}\left(t_{f}\right)=\xi_{2}\left(t_{f}\right) & \gamma_{11-16}\left(t_{f}\right)=0 \\
\gamma_{3}\left(t_{f}\right)=\xi_{1}\left(t_{f}\right) & &
\end{array}
$$

The values in eq. (28) represent the real physical values of the tracked paths.

\section{COMPUTATION AND RESULTS}

\subsection{Computation}

The results of the path tracking algorithm were computed for an effective mass of $m=4 \cdot 10^{5} \mathrm{~kg}$, cylinder areas $A_{A}=A_{B}=6 \cdot 10^{-2} \mathrm{~m}^{2}$, friction parameter $b=1.4 \cdot 10^{7} \frac{\mathrm{NS}}{\mathrm{m}}$ and a bulk modulus of $\beta=1.4 \cdot 10^{9} \mathrm{~Pa}$. The value for the friction parameter $b$ was identified during a cutting experiment. Furthermore, the values for the linearized terms $C_{q 1}, C_{q 2}, C_{p 1}$ and $C_{p 2}$ were calculated for the supply pressure of $300 \cdot 10^{5} \mathrm{~Pa}$ and it is assumed that the initial values for the pressures on the both sides will be $10 \cdot 10^{5} \mathrm{~Pa}$. An oblique rectangular shape was chosen as the reference path for the pressure. This increased the overall stiffness of the system during the motion. For the position we have chosen a path that is followed by one of the cutting arms of the real machine. Due to the different dimensions and magnitudes of the two tracked states, the values of the normalization parameter $\mu_{2}$ was assumed to be $\mu_{2}=10^{4}$. The values of $\mu_{3}$ and $\mu_{4}$ were experimentally determined to be $5 \cdot 10^{-5}$. The path tracking algorithm was firstly computed in MATLAB, where the control variables $u_{1}$ and $u_{2}$ were calculated. Then the system presented in fig.

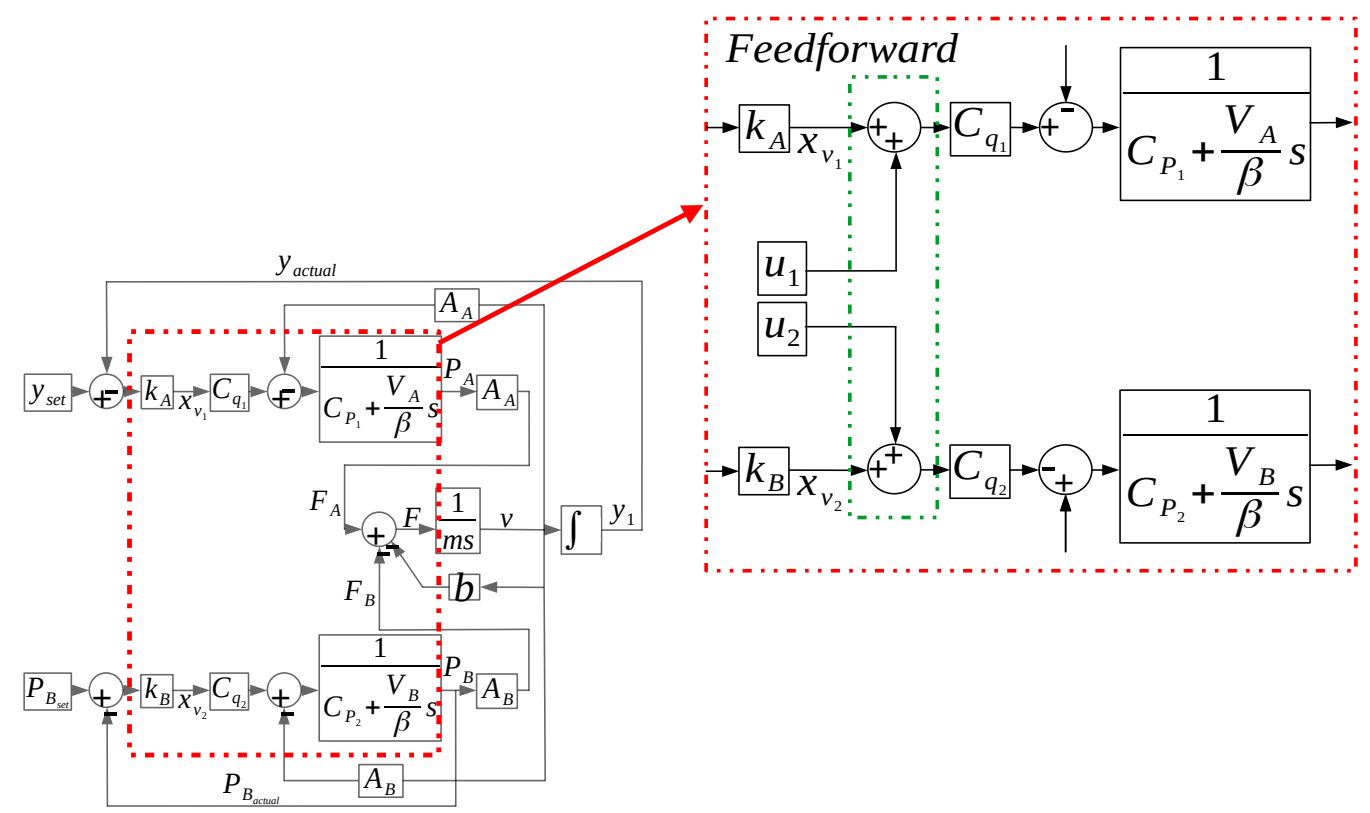

Figure 3: Implementation of the calculated control variables $u_{1}$ and $u_{2}$ into the simulation model of the system as a feed-forward.

2 was simulated in MATLAB Simulink where the control variables were inserted as feed forward inputs to the system (see fig. 3).

\footnotetext{
${ }^{1}$ Due to the fact that some of the conditions are on the Lagrangian multiplier $\lambda$, only the conditions for the control variables need to be defined.
} 


\subsection{Results}

The system presented in fig. 1 was simulated for two different time intervals, namely for $t \in[0,4] s$ and for $t \in[0,8] s$. In fig. 4 it can be seen that the system is following the given trajectories smoothly and precisely. The
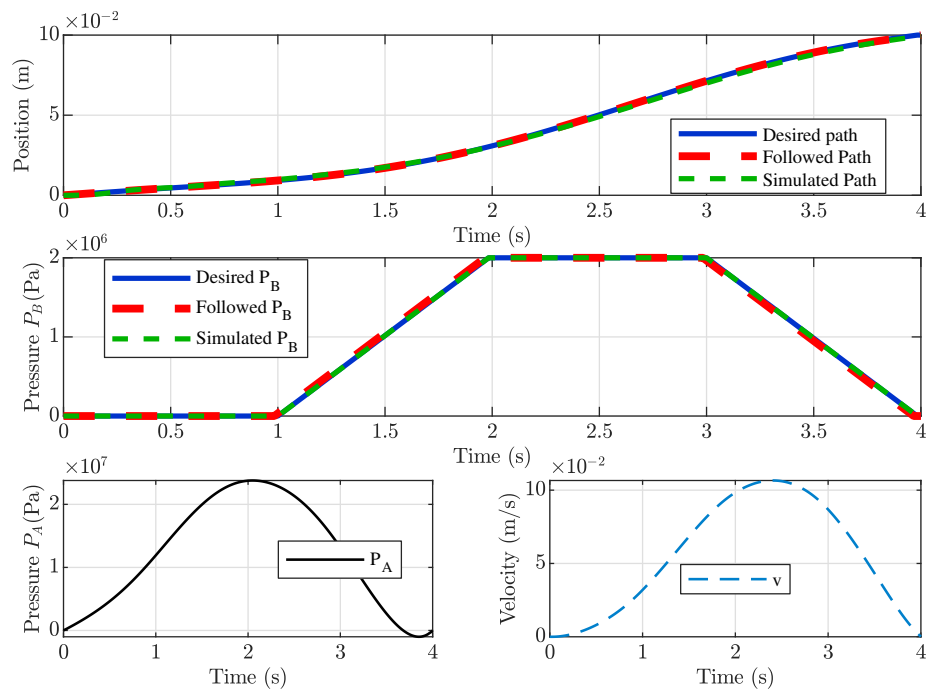

Figure 4: (Top and middle graph) - Results of the path tracking algorithm for time $t \in[0,4] s$. The states $x_{3}$ and $x_{2}$ (position and pressure respectively) are being tracked. The followed values and the simulated values deviate only slightly from the desired values. (Bottom graphs) - The behavior of the remaining two states of the system.

maximum value of the pressure on the rod side is set to $20 \cdot 10^{5} \mathrm{~Pa}$. This increases the mechanical stiffness of the hydraulic system which will increase the mechanical stiffness of the overall system. Although the pressure

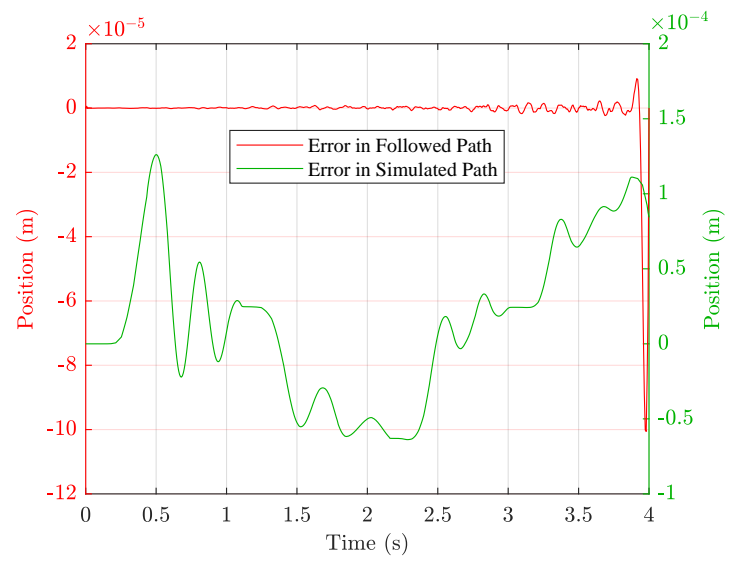

(a) Error curves for the followed and simulated positions in fig. 4 .

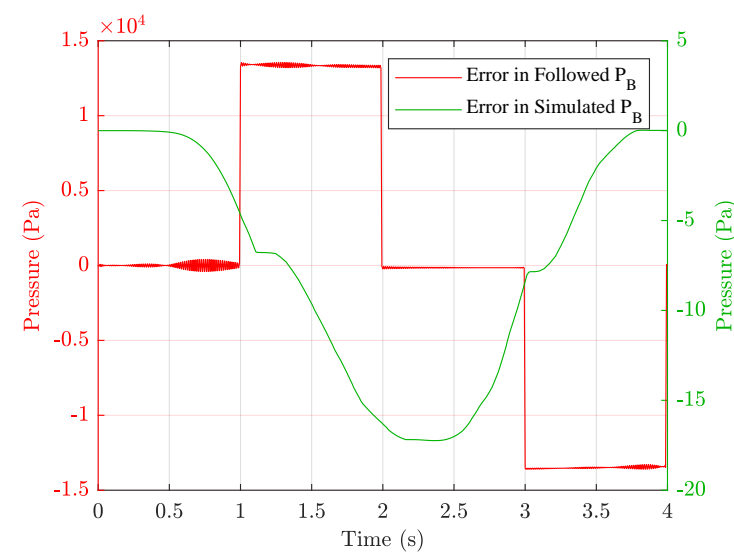

(b) Error curves for the followed and simulated pressures in fig. 4 .

Figure 5: Error curves for the followed and simulated positions (left graph) and pressures (right graph) in fig. 4 accordingly. The maximum offset for the path tracking method is smaller than $1 \%$.

changes frequently during the time interval, there are no oscillations in the solution. This is not the case when using conventional controllers. The same applies for the position. The system reaches its maximum speed near the middle point of the path, demonstrating the energy efficient performance of this method. To be able to observe the accuracy of the new method, the error was observed for both the position and the pressure in fig. 5. It can be seen that the maximum offset for the position and the pressure is smaller $0.01 \%$ and $1 \%$ accordingly. This shows that the path tracking method has a very high accuracy. On the other hand in fig. 6, the system follows the same path for position and pressure but in double the time. Accordingly, the pressure on the piston side and the velocity are much smaller than in fig. 4. From fig. 7 it can be seen that the path tracking method shows even higher accuracy for longer paths. The maximum errors are in the magnitude of $0.005 \%$. 

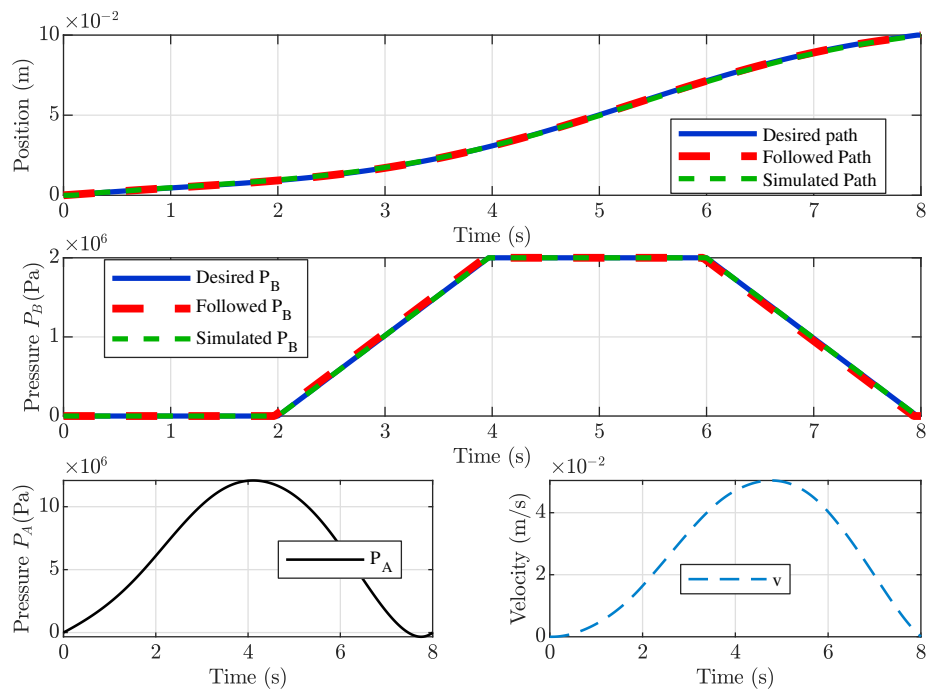

Figure 6: (Top and middle graph) - Results of the path tracking algorithm for time $t \in[0,8] s$. The states $x_{3}$ and $x_{2}$ (position and pressure respectively) are being tracked. (Bottom graphs) - The system is tracking the same distance over a longer period of time, which results in smaller pressure values on the piston side and a decreased velocity.

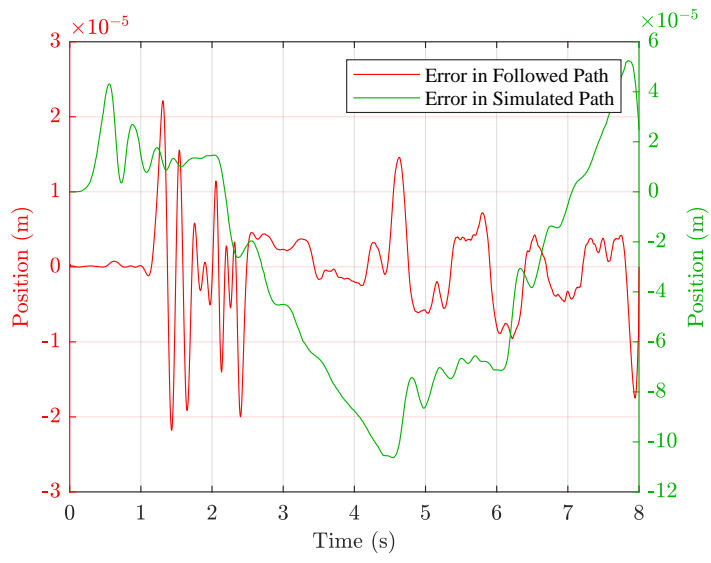

(a) Error curves for the followed and simulated positions in fig. 6

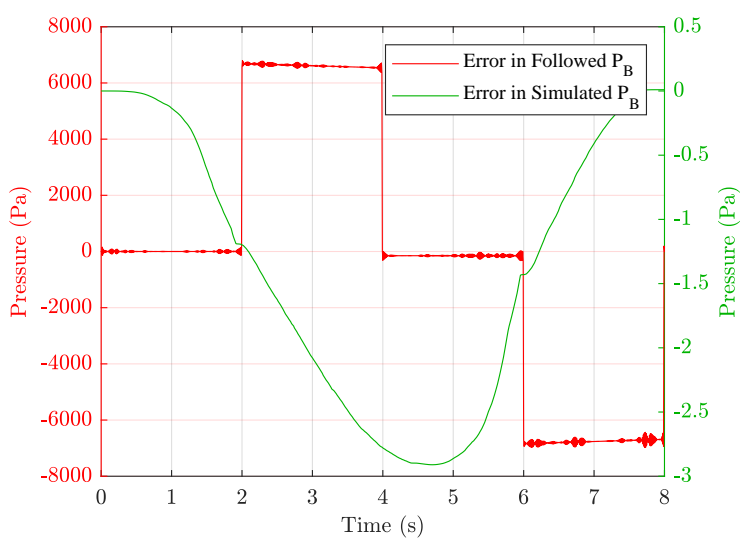

(b) Error curves for the followed and simulated pressures in fig. 6

Figure 7: Error curves for the followed and simulated positions and pressures in fig. 6 accordingly. Here the path tracking method shows maximum offset of $0.005 \%$.

\section{CONCLUSIONS AND FUTURE WORKS}

\subsection{Conclusions}

From the results obtained it can be concluded that the path tracking method can be used successfully to track states in different units and of different magnitudes. The calculated control variables, which were later used as feed forward inputs for the simulated system, provided paths with very small offset from the desired and the followed ones. Furthermore, the behavior of the other two states shows that the method is energy efficient for both tested scenarios.

\subsection{Future Works}

In this paper we use the mass matrix method and the interstitial derivatives to find stable numerical solution to a stiff numerical problem. Further we have implemented only P (proportional part of PID) controllers on the pressure and flow controlled side. In practice however, the valves have also I (integral part of PID) controllers. The numerical solution of the integro-differential equations for these systems could be a question of future work. Additionally, the further implementation of this method on the real system is of great interest. 


\section{References}

[1] H.J. Sussmann and J.C. Willems. 300 years of optimal control: from the brachystochrone to the maximum principle. IEEE Control Systems, 17(3):32-44, 1997.

[2] Gerhard Rath and Emil Zaev. Optimal Control for Hydraulic System With Separate Meter-in and Separate Meter-Out. The 15th Scandinavian International Conference on Fluid Power, SICFP'17, June 7-9, 2017, Linköping, Sweden, pages 125-134, 2017.

[3] Randal W. Bea. Successive Galerkin approximation algorithms for nonlinear optimal and robust control. International Journal of Control, 71(5):717-743, 1998.

[4] B Eriksson and J-O Palmberg. Individual metering fluid power systems: challenges and opportunities. Proceedings of the Institution of Mechanical Engineers, Part I: Journal of Systems and Control Engineering, 225(2):196-211, 2011.

[5] G Rath, M Harker, and E Zaev. Direct numerical solution of stiff ODE systems in optimal control. In 2017 6th Mediterranean Conference on Embedded Computing (MECO), pages 1-5, 2017.

[6] Lawrence F. Shampine and Mark W. Reichelt. The MATLAB ode suite, volume 18. 1997.

[7] B.J. Alshaer, T.T. Darabseh, and M.A. Alhanouti. Path planning, modeling and simulation of an autonomous articulated heavy construction machine performing a loading cycle. Applied Mathematical Modelling, 37(7):5315-5325, 2013.

[8] B.J. Alshaer, T.T. Darabseh, and A.Q. Momani. Modelling and control of an autonomous articulated mining vehicle navigating a predefined path. International Journal of Heavy Vehicle Systems, 21(2):152, 2014.

[9] Xuewu Ji, Yulong Liu, Xiangkun He, Kaiming Yang, Xiaoxiang Na, Chen Lv, and Yahui Liu. Interactive Control Paradigm-Based Robust Lateral Stability Controller Design for Autonomous Automobile Path Tracking With Uncertain Disturbance: A Dynamic Game Approach. IEEE Transactions on Vehicular Technology, 67(8):6906-6920, 2018.

[10] Hans Andersen, Zhuang Jie Chong, You Hong Eng, Scott Pendleton, and Marcelo H. Ang. Geometric path tracking algorithm for autonomous driving in pedestrian environment. In 2016 IEEE International Conference on Advanced Intelligent Mechatronics (AIM), pages 1669-1674. IEEE, 2016.

[11] Guoxing Bai, Li Liu, Yu Meng, Weidong Luo, Qing Gu, and Baoquan Ma. Path tracking of mining vehicles based on nonlinear model predictive control. Applied Sciences (Switzerland), 9(7), 2019.

[12] Jie Ji, Amir Khajepour, Wael William Melek, and Yanjun Huang. Path Planning and Tracking for Vehicle Collision Avoidance Based on Model Predictive Control With Multiconstraints. IEEE Transactions on Vehicular Technology, 66(2):952-964, 2017.

[13] J M Maciejowski. Predictive Control with Constraints. Prentice Hall, England., 2002.

[14] Tao Wang, Lianyu Zhao, Yunhui Jia, and Jutao Wang. Robot Path Planning Based on Improved Ant Colony Algorithm. In 2018 WRC Symposium on Advanced Robotics and Automation (WRC SARA), pages 70-76. IEEE, 2018.

[15] G. Kalaiarassan and K. Krishnamurthy. Digital hydraulic single-link trajectory tracking control through flowbased control. Measurement and Control (United Kingdom), 52(7-8):775-787, 2019.

[16] Morten H. Rudolfsen, Teodor N. Aune, Oddgeir Auklend, Leif Tore Aarland, and Michael Ruderman. Identification and Control Design for Path Tracking of Hydraulic Loader Crane. IEEE/ASME International Conference on Advanced Intelligent Mechatronics, AIM, pages 565-570, 2017.

[17] Johannes Handler, Matthew Harker, and Gerhard Rath. Multidimensional Path Tracking With Global Least Squares Solution. 21 st IFAC World Congress, 21, 2020. 
[18] Jie Wang, Min Cheol Lee, Karam Dad Kallu, Saad Jamshed Abbasi, and Seokyoung Ahn. Trajectory tracking control of a hydraulic system using TSMCSPO based on sliding perturbation observer. Applied Sciences (Switzerland), 9(7):1-17, 2019.

[19] Jih Hua Chin, Yen His Sun, and Yuan Ming Cheng. Force computation and continuous path tracking for hydraulic parallel manipulators. Control Engineering Practice, 16(6):697-709, 2008.

[20] Bin Zhang, Shuang Wang, Yuting Liu, and Huayong Yang. Research on Trajectory Planning and Autodig of Hydraulic Excavator. Mathematical Problems in Engineering, 2017:1-10, 2017.

[21] Seonhyeok Kang, Jaemann Park, Seunghyun Kim, Bongju Lee, Youngbum Kim, Panyoung Kim, and H. Jin Kim. Path tracking for a hydraulic excavator utilizing proportional-derivative and linear quadratic control. In 2014 IEEE Conference on Control Applications (CCA), pages 808-813. IEEE, 2014.

[22] Goran Stojanoski, Gerhard Rath, and Martin Gimpel. The Effects of Bulk Modulus on the Dynamics of Hydraulic Independet Metering Systems. Sixteenth Scandinavian International Conference on Fluid Power, pages $276-290,2019$.

[23] Y F Liu, J Li, Z M Zhang, X H Hu, and W J Zhang. Experimental comparison of five friction models on the same test-bed of the micro stick-slip motion system. pages 15-28, 2015.

[24] Herbert E. Merrit. Hydraulic Control Systems. John Wiley \& Sons, Inc., 1967.

[25] Peter Chapple. Principles of Hydraulic Systems Design. Momentum Press, vol. 2 edition, 2015.

[26] Richard Belman. Mathematical Optimization Techniques. 1963.

[27] I M Gelfand and S V Fomin. Calculus of Variations. Dover Books on Mathematics. Dover Publications, 2012.

[28] Matthew Harker and Gerhard Rath. Discrete Inverse Problem Approach to Path Tracking in State Space Form. In 2018 International Conference on Applied Electronics (AE), pages 1-4. IEEE, 2018.

[29] Matthew Harker. Fractional Differential Equations: Numerical Methods for Applications. Springer International Publishing, 2020. 